\title{
INDEX TO VOLUME 13
}

Arbarello, Enrico, Krichever, Igor, and Marini, Giambattista Characterizing Jacobians via flexes of the Kummer Variety ...................

Baker, Matthew A lower bound for average values of dynamical Green's functions .......................................... 245

Balduzzi, David Donagi-Markman cubic for Hitchin systems ........... 923

Banks, William D., and Shparlinski, Igor E. Short character sums with Beatty sequences ...................................... 539

Baryshnikov, Yuliy, and Zharnitsky, Vadim Sub-Riemannian geometry and periodic orbits in classical billiards ....................... 587

Beck, Matthias, and Hoşten, Serkan Cyclotomic polytopes and growth series of cyclotomic lattices ................................. 607

Benedetto, John J., Czaja, Wojciech, Powell, Alexander M., and Sterbenz, Jacob An Endpoint $(1, \infty)$ Balian-Low Theorem .......... 467

Bierstone, Edward, Fefferman, Charles, Milman, Pierre D., and Pawlucki, Wieslaw Examples concerning Whitney's $C^{m}$ extension prob-

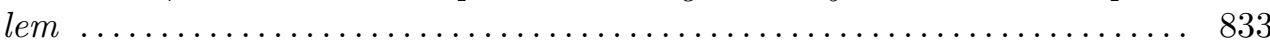

Bourgeois, Frédéric Contact homology and homotopy groups of the space of contact structures .................................... 71

Bove, Antonio, Derridj, Makhlouf, Kohn, Joseph J., and Tartakoff, David S. Sums of squares of complex vector fields and (analytic-) hypoellipticity ....................................... 683

Boyer, Charles P., and Galicki, Krzysztof Erratum and Addendum for "Rational homology 5-spheres with positive Ricci curvature" ............

Brînzănescu, Vasile, and Moraru, Ruxandra Twisted Fourier-Mukai transforms and bundles on non-Kähler elliptic surfaces ............. 501

Budur, Nero, Mustaţă, Mircea, and Saito, Morihiko Roots of BernsteinSato polynomials for monomial ideals: a positive characteristic approach . 125

Burman, Yurii, and Shapiro, Boris Around matrix-tree theorem ....... 761

Caicedo, Andrés Eduardo, and Veličković, Boban The bounded proper forcing axiom and well orderings of the reals ................... 393

Catanese, Fabrizio, Chen, Meng, and Zhang, De-Qi The Noether inequality for smooth minimal 3-folds ........................... 653

Chen, Meng see Catanese, Fabrizio, Chen, Meng, and Zhang,

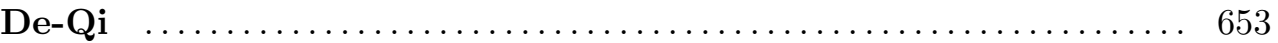

Colombini, Ferruccio, Grammatico, Cataldo, and Tataru, Daniel Strong uniqueness for second order elliptic operators with Gevrey coefficients ...................................... 15

Conway, J. H. see Rossetti, J. P., and Conway, J. H. ............. 475

Czaja, Wojciech see Benedetto, John J., Czaja, Wojciech, Powell, Alexander M., and Sterbenz, Jacob 
Danielli, Donatella, and Petrosyan, Arshak Full regularity of the free boundary in a Bernoulli-type problem in two dimensions ............. 667

Derridj, Makhlouf see Bove, Antonio, Derridj, Makhlouf, Kohn, Joseph J., and Tartakoff, David S. .................... 683

Dieulefait, Luis V. Galois characterization of Endoscopy for rational Siegel modular forms ..................................... 317

Dynkin, E. B. On extreme X-harmonic functions ................. 59

Enriquez, Benjamin On the Drinfeld generators of $\mathfrak{g r t}_{1}(\mathbf{k})$ and $\Gamma$-functions

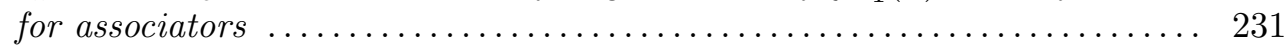

Escauriaza, L., Kenig, C.E., Ponce, G., and Vega, L. Decay at infinity of caloric functions within characteristic hyperplanes ............... 441

Fefferman, Charles see Bierstone, Edward, Fefferman, Charles, Milman, Pierre D., and Pawlucki, Wieslaw ................ 833

Fel'shtyn, Alexander, Troitsky, Evgenij, and Vershik, Anatoly Twisted Burnside theorem for type $I I_{1}$ groups: an example ................. 719

Fernando, José F., Ruiz, Jesús M., and Scheiderer, Claus Sums of squares of linear forms ................................. 947

Frigerio, Roberto, and Martelli, Bruno Countable groups are mapping class groups of hyperbolic 3 -manifolds ...................... 897

Galicki, Krzysztof see Boyer, Charles P., and Galicki, Krzysztof _. 463

Garnett, John, Prat, Laura, and Tolsa, Xavier Lipschitz harmonic capacity and bilipschitz images of Cantor sets ................... 865

Gee, Toby A modularity lifting theorem for weight two Hilbert modular

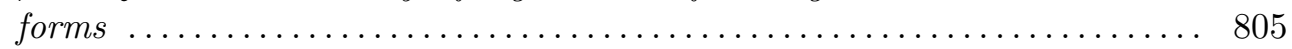

Ghitza, Alexandru All Siegel Hecke eigensystems (mod p) are cuspidal ... 813

Gholampour, Amin, and Song, Yinan Evidence for the Gromov-Witten/Donaldson-Thomas Correspondence ............... 623

Goldstein, Edward Area comparison results for isotropic surfaces ....... 333

Gordon, Carolyn, and Perry, Peter Continuous families of isophasal scattering manifolds ....................................... 631

Grammatico, Cataldo see Colombini, Ferruccio, Grammatico, Cataldo,

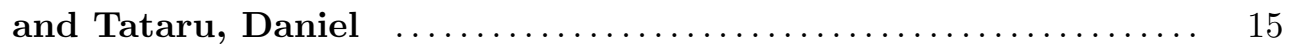

Gressman, Philip T. $L^{p}$-improving properties of X-ray like transforms $\ldots 787$

Guerzhoy, P. On a conjecture of Atkin for the primes 13,17,19, and 23 . . 549

Gustafson, Stephen, Nakanishi, Kenji, and Tsai, Tai-Peng Scattering for the Gross-Pitaevskii equation ............................ 273

Hara, Nobuo, with an appendix by Paul Monsky F-pure thresholds and F-jumping exponents in dimension two ..................... 747

Hoşten, Serkan see Beck, Matthias, and Hoşten, Serkan ........ 607

$\mathbf{H u}$, Wei, and $\mathbf{X i}$, Changchang Auslander-Reiten sequences and global di-

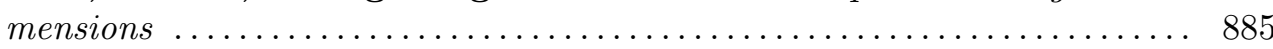

Huang, Xiaojun, Ji, Shanyu, and Xu, Dekang A new gap phenomenon for proper holomorphic mappings from $B^{n}$ into $B^{N} \ldots \ldots \ldots \ldots \ldots \ldots .515$

Huybrechts, Daniel, and Thomas, Richard $\mathbb{P}$-objects and autoequivalences of derived categories .................................... 87 
Ivanov, Stefan, Parton, Maurizio, and Paccinni, Paolo Locally conformal parallel $G_{2}$ and Spin(7) manifolds

Jakab, Tünde, and Mitrea, Marius Parabolic initial boundary value problems in nonsmooth cylinders with data in anisotropic Besov spaces ..... 825

Ji, Shanyu see Huang, Xiaojun, Ji, Shanyu, and Xu, Dekang ..... 515

Kaledin, D. On the coordinate ring of a projective Poisson scheme ....... 99

Kenig, C.E. see Escauriaza, L., Kenig, C.E., Ponce, G., and

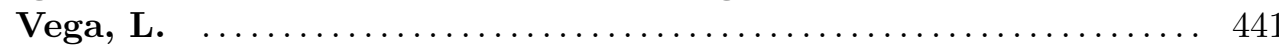

Kisilevsky, Hershy, and Sonn, Jack Abelian extensions of global fields with constant local degree ....................................... 599

Kohn, Joseph J. see Bove, Antonio, Derridj, Makhlouf, Kohn, Joseph J., and Tartakoff, David S. ........................ 683

Krichever, Igor see Arbarello, Enrico, Krichever, Igor, and Marini,

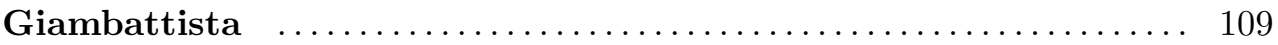

Lam, Thomas A combinatorial generalization of the Boson-Fermion correspondence ......................................... 377

Lee, Sangyop Reducing and toroidal Dehn fillings on 3-manifolds bounded by two tori ............................................... 287

Levin, Aaron One-parameter families of unit equations ............... 935

Li, Yi Some Results of the Mariño-Vafa Formula ................... 847

Luijk, Ronald van Quartic K3 surfaces without nontrivial automorphisms . 423

Luzzatto, Stefano, and Wang, Lanyu Topological invariance of generic non-uniformly expanding multimodal maps .................... 343

Marini, Giambattista see Arbarello, Enrico, Krichever, Igor, and Marini, Giambattista ............................. 109

Martelli, Bruno see Frigerio, Roberto, and Martelli, Bruno ...... 897

Mateu, Joan, and Verdera, Joan $L^{p}$ and weak $L^{1}$ estimates for the maximal Riesz transform and the maximal Beurling transform .............. 957

Mermin, Jeffrey, and Peeva, Irena Lexifying ideals ............... 409

Milas, Antun Modular invariance, modular identities and supersingular $j$ invariants ........................................ 729

Milman, Pierre D. see Bierstone, Edward, Fefferman, Charles, Milman, Pierre D., and Pawlucki, Wieslaw ............... 833

Miranda, Eva, and Zung, Nguyen Tien A note on equivariant normal forms of Poisson structures ................................ 1001

Mitrea, Marius see Jakab, Tünde, and Mitrea, Marius _......... 825

Monniaux, Sylvie Navier-Stokes equations in arbitrary domains: the FujitaKato scheme .................................... 455

Moraru, Ruxandra see Brînzănescu, Vasile, and Moraru, Ruxandra 501

Mustaţă, Mircea see Budur, Nero, Mustaţă, Mircea, and Saito, Morihiko ....................................... 125

Nakanishi, Kenji see Gustafson, Stephen, Nakanishi, Kenji, and Tsai,

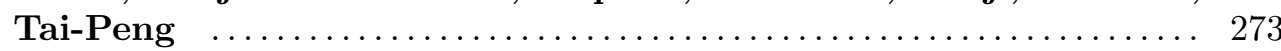

Nazarov, F., Volberg, A., and Yuditskii, P. Asymptotics of orthogonal polynomials via the Koosis theorem ............................ 975

Oguiso, Keiji Tits alternative in hypekähler manifolds .............. 307 
Owens, Brendan $A$ characterisation of the $n\langle 1\rangle \oplus\langle 3\rangle$ form and applications to rational homology spheres .......................... 259

Paccinni, Paolo see Ivanov, Stefan, Parton, Maurizio, and Paccinni, Paolo

Papikian, Mihran On the torsion of optimal elliptic curves over function

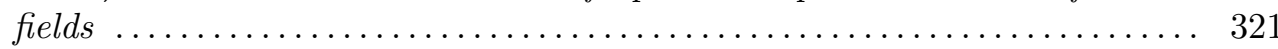

Parton, Maurizio see Ivanov, Stefan, Parton, Maurizio, and Paccinni,

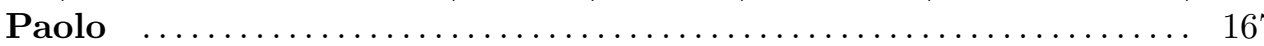

Pawlucki, Wieslaw see Bierstone, Edward, Fefferman, Charles, Milman, Pierre D., and Pawlucki, Wieslaw $\ldots \ldots \ldots \ldots \ldots \ldots \ldots . \ldots 33$

Payne, Sam Equivariant Chow cohomology of toric varieties . . . . . . . . 29

Peeva, Irena see Mermin, Jeffrey, and Peeva, Irena . . . . . . . . . 409

Peherstorfer, F., Volberg, A., and Yuditskii, P. Limit periodic Jacobi matrices with a prescribed p-adic hull and a singular continuous spectrum 215

Perry, Peter see Gordon, Carolyn, and Perry, Peter ........... 631

Petrosyan, Arshak see Danielli, Donatella, and Petrosyan, Arshak . 667

Plamenevskaya, Olga Transverse knots and Khovanov homology ........ 571

Ponce, G. see Escauriaza, L., Kenig, C.E., Ponce, G., and Vega, L. 441

Powell, Alexander M. see Benedetto, John J., Czaja, Wojciech, Powell, Alexander M., and Sterbenz, Jacob ............... 467

Prat, Laura see Garnett, John, Prat, Laura, and Tolsa, Xavier . . . 865

Rietsch, K. Closure relations for totally nonnegative cells in $G / P \ldots \ldots 775$

Robert, O., and Sargos, P. A general bound for oscillatory integrals with a

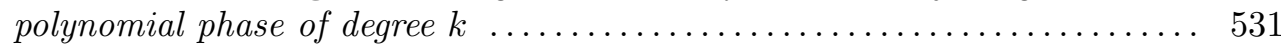

Rogers, Keith M. On a planar variant of the Kakeya problem . . . . . . . . 199

Rohrlich, David E. Root numbers of semistable elliptic curves in division

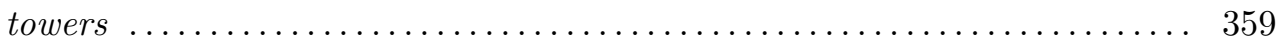

Rossetti, J. P., and Conway, J. H. Hearing the Platycosms . . . . . . . . 475

Ruiz, Jesús M. see Fernando, José F., Ruiz, Jesús M., and Scheiderer,

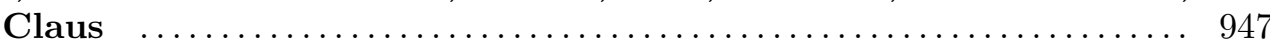

Saito, Morihiko see Budur, Nero, Mustaţă, Mircea, and Saito, Mori-

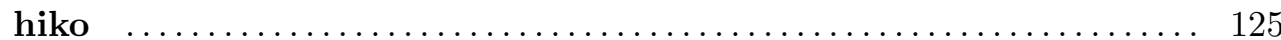

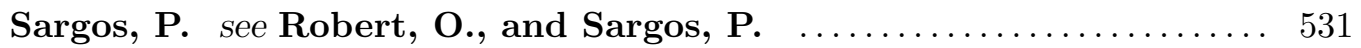

Saïd, Salem Ben Huygens' principle for the wave equation associated with the trigonometric Dunkl-Cherednik operators ....................

Scheiderer, Claus see Fernando, José F., Ruiz, Jesús M., and Schei-

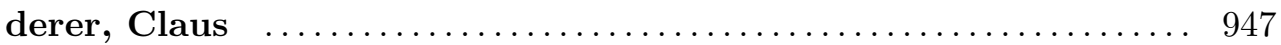

Seo, Soogil Circular distributions of finite order ................. 1

Seshadri, Harish Negative sectional curvature and the product complex struc-

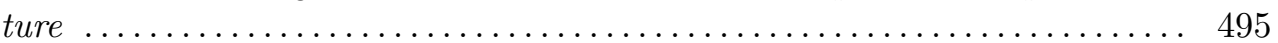

Shapiro, Boris see Burman, Yurii, and Shapiro, Boris _........ 761

Shen, Zhongwei The $L^{p}$ Dirichlet problem for elliptic systems on Lipschitz

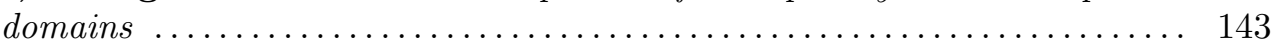

Shparlinski, Igor E. see Banks, William D., and Shparlinski, Igor E. 539

Smith, Hart F. Sharp $L^{2} \rightarrow L^{q}$ bounds on spectral projectors for low regularity

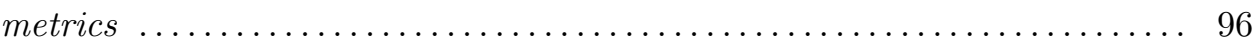


Song, Yinan see Gholampour, Amin, and Song, Yinan ......... 623

Sonn, Jack see Kisilevsky, Hershy, and Sonn, Jack ............. 599

Sterbenz, Jacob see Benedetto, John J., Czaja, Wojciech, Powell, Alexander M., and Sterbenz, Jacob $\ldots \ldots \ldots \ldots \ldots \ldots \ldots \ldots 467$

Street, Brian $L^{p}$ regularity for Kohn's operator .................. 703

Tartakoff, David S. see Bove, Antonio, Derridj, Makhlouf, Kohn, Joseph J., and Tartakoff, David S. ................... 683

Tataru, Daniel see Colombini, Ferruccio, Grammatico, Cataldo, and

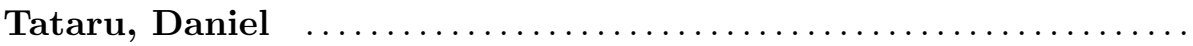

Taubes, Clifford Henry A proof of a theorem of Luttinger and Simpson about the number of vanishing circles of a near-symplectic form on a 4-dimensional manifold ................................ 557

Thomas, Richard see Huybrechts, Daniel, and Thomas, Richard $\ldots 87$

Thomas, Teruji The Maslov index as a quadratic space ............... 985

Tolsa, Xavier see Garnett, John, Prat, Laura, and Tolsa, Xavier . . 865

Troitsky, Evgenij see Fel'shtyn, Alexander, Troitsky, Evgenij, and Vershik, Anatoly ................................... 719

Tsai, Tai-Peng see Gustafson, Stephen, Nakanishi, Kenji, and Tsai,

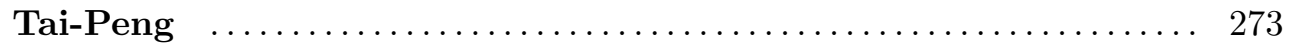

Vega, L. see Escauriaza, L., Kenig, C.E., Ponce, G., and Vega, L. .. 441

Verdera, Joan see Mateu, Joan, and Verdera, Joan _............. 957

Vershik, Anatoly see Fel'shtyn, Alexander, Troitsky, Evgenij, and Vershik, Anatoly ................................. 719

Volberg, A. see Peherstorfer, F., Volberg, A., and Yuditskii, P. . . 215

Volberg, A. see Nazarov, F., Volberg, A., and Yuditskii, P. ....... 975

Wang, Lanyu see Luzzatto, Stefano, and Wang, Lanyu .......... 343

Wehrheim, Katrin Energy identity for anti-self-dual instantons on $\mathbb{C} \times \Sigma$. 161

Wingberg, Kay On Čebotarev Sets ................................ 179

Wuthrich, Christian Extending Kato's result to elliptic curves with p-isogenies ...................................... 713

Xi, Changchang see Hu, Wei, and Xi, Changchang ............. 885

$\mathbf{X u}$, Dekang see Huang, Xiaojun, Ji, Shanyu, and Xu, Dekang . . . 515

Yau, Stephen T., and Zhang, Letian An upper estimate of integral points in real simplices with an application to singularity theory ............ 911

Yuditskii, P. see Peherstorfer, F., Volberg, A., and Yuditskii, P. . . 215

Yuditskii, P. see Nazarov, F., Volberg, A., and Yuditskii, P. ...... 975

Zhang, De-Qi see Catanese, Fabrizio, Chen, Meng, and Zhang,

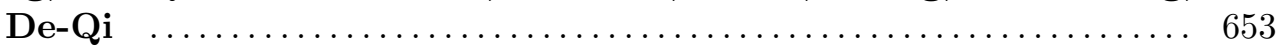

Zhang, Letian see Yau, Stephen T., and Zhang, Letian .......... 911

Zharnitsky, Vadim see Baryshnikov, Yuliy, and Zharnitsky, Vadim 587

Zung, Nguyen Tien see Miranda, Eva, and Zung, Nguyen Tien .... 1001

Veličković, Boban see Caicedo, Andrés Eduardo, and Veličković, Boban ............................................. 393 